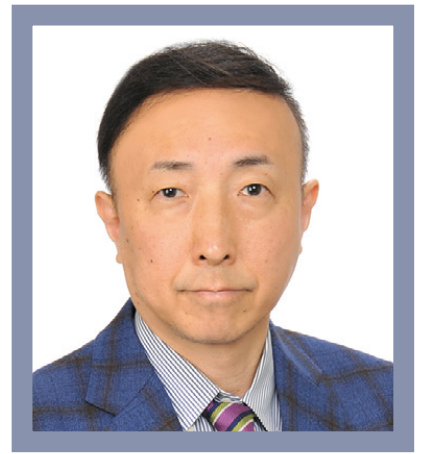

\title{
23rd Annual Scientific Meeting of the International Society of Cardiovascular Pharmacotherapy
}

\author{
Koji Hasegawa
}

President, International Society of Cardiovascular Pharmacotherapy (ISCP)

Chair, 23rd Annual Scientific Meeting of the ISCP in Kyoto, Japan

Citation: European Cardiology Review 2018;13(2):119 DoI: https://doi.org/10.15420/ecr.2018.13.2.GE2

Correspondence: Koji Hasegawa, National Hospital Organization, Kyoto Medical Center, Kyoto, Japan. E: koj@kuhp.kyoto-u.ac.jp

$\mathrm{C}$ ardiovascular pharmacotherapy has drastically advanced in the past 30 years and made a major contribution to health and welfare worldwide. This advancement is attributable to the accumulation of evidence from clinical trials. Blood pressure and lipid management is the most important first-line therapy for arteriosclerotic cardiovascular disease. The medical treatment of systolic heart failure has drastically improved the 5-year survival rate. Prevention of cerebral embolism in AF has been achieved by the wide distribution of novel oral anticoagulants.

The development of pharmacotherapy has greatly benefited patients with heart and vessel diseases. However, cardiovascular diseases remain the most serious cause of death worldwide, with some 18 million lives lost annually because of them. Educating medical workers on risk management by pharmacotherapy, based on guidelines, is an important social contribution. In addition, recent advances in basic science have elucidated pathophysiology and signal transduction mechanisms for the onset of heart and vessel diseases. Further benefits could be achieved for patients with cardiovascular disease if we applied information obtained from basic research to clinical situations.
The International Society of Cardiovascular Pharmacotherapy (ISCP) promotes research on pharmacotherapy for cardiovascular diseases. The ISCP introduced cardiovascular pharmacotherapy reviews, international clinical trials results and pharmaceutical safety information. We disseminate this knowledge through our website and scientific meetings.

The 23rd Annual Scientific Meeting of the ISCP was held in Kyoto, Japan, on 26 and 27 May 2018. Medical workers, including physicians and pharmacists, attended educational seminars and received training about appropriate cardiovascular pharmacotherapy. An active discussion was conducted for optimal cardiovascular pharmacotherapy based on evidence. In poster sessions of general abstracts, young investigators presented results of basic and translational research for future cardiovascular pharmacotherapy. In the following section, we present some of the excellent abstracts.

We are eagerly seeking more improvement in prognosis for patients with heart and vessel diseases through future development of cardiovascular pharmacotherapy. 